\title{
Cyclicities in the light variations of S Doradus stars
}

\section{P Cygni}

\author{
M. de Groot $^{1}$, C. Sterken ${ }^{2, \star}$, and A. M. van Genderen ${ }^{3}$ \\ 1 Armagh Observatory, College Hill, Armagh BT61 9DG, Northern Ireland \\ 2 University of Brussels (VUB), Pleinlaan 2, 1050 Brussels, Belgium \\ 3 Leiden Observatory, Postbus 9513, 2300 RA Leiden, The Netherlands
}

Received 19 April 2001 / Accepted 2 July 2001

\begin{abstract}
On the basis of new photometric observations and archived data published since 1907, we discuss the light variations of $\mathrm{P}$ Cygni. We conclude that there are $\alpha$ Cygni-type microvariations with a stable (pulsation) quasi-period of 17.3 days. There are also longer cycles of variation with $P \sim 100 \mathrm{~d}$, so-called 100 d-type microvariations, and with $P \sim 1500-1600$ d, a short S Dor-type phase.
\end{abstract}

Key words. stars: individual: P Cygni - stars: variables - stars: oscillations - stars: supergiants

\section{Introduction}

This is the third detailed study on cyclicities in the light variations of a selection of well-monitored S Doradus variables. The two previous papers dealt with $\zeta^{1}$ Sco in the Galaxy and R 40 in the SMC (Sterken et al. 1997a, 1998, respectively).

$\mathrm{S}$ Doradus stars - also known as Luminous Blue Variables (LBVs) - are found in the upper left-hand corner of the Hertzsprung-Russell diagram. They are photometrically variable with a large range of amplitudes (several hundredths of a magnitude to magnitudes) and on a vast range of time scales (hours, over decades, to centuries). The amplitudes of the variations seem to increase with the time scales at which they occur. Considering the presence of circumstellar ejecta, about $40 \%$ of the S Dor stars seem to have suffered an $\eta$ Carinae-type outburst in the past. For an extensive review of the light curve properties of S Dor stars, we refer to van Genderen (2001), who classified P Cyg as a weak-active (w-a) S Dor variable (because it was weak-active in the 20th century).

P Cygni (HR 7763 = HD 193237) is a notorious S Dor star of the $\eta$ Carinae type, with giant eruption(s), $\mathrm{S}$ Doradus phases and microvariability (for a detailed discussion, see de Groot 1969, and for a recent review see Israelian \& de Groot 1999). According to Stothers (1999a),

Send offprint requests to: C. Sterken,

e-mail: csterken@vub.ac.be

* Research Director, Belgian Fund for Scientific Research (FWO).
P Cyg is in a "state of marginal dynamical instability" and de Jager (2001), studying its photosphere, used the following expression: "it finds itself at most at the fringe of instability". Dynamical instability means that the outer layers are subject to a steady expansion or contraction, and in the first case eject matter vigorously. A discussion of the periodicity of the radial velocity and light variations of P Cyg was given by van Gent \& Lamers (1986), see also van Genderen (1991) and van Genderen et al. (1992). A long time scale spectroscopic study of the $\mathrm{H} \alpha$ emission line was compared with simultaneous photometry from different sources by Markova $(2000,2001)$ and Markova et al. (2001a, 2001b). These authors found various correlations with different time scales between the equivalent width of the $\mathrm{H} \alpha$ line and the photometric behaviour. This is the first time that such a study of an S Dor variable has been made.

$\mathrm{P}$ Cygni is one of the very few hypergiants that have a well-documented light history. Discovered by Willem Jansz Blaeu on 18th August 1600 as a third-magnitude star, it was only the second so-called "nova" after Tycho's "new" star of 1572. This, and the invention of the telescope at about the same time, ensured a long series of observations of P Cygni, starting in 1600 and still continuing today. Though the observations of the first three centuries suffer from the fact that they were done visually, by different observers using different comparison stars, and in the absence of an agreed magnitude scale, it has still been possible to recover P Cygni's photometric history. An investigation of the characteristics of the light curve 


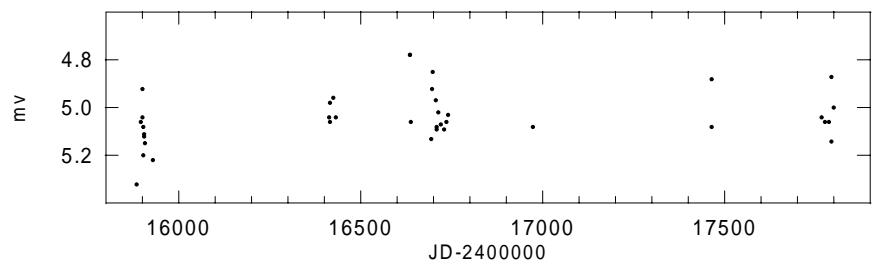

Fig. 1. $m_{v}$ light curve of $\mathrm{P}$ Cyg, data from von Prittwitz (1907).

after 1700 has led to the conclusion that we are witnessing photometric changes due to stellar evolution (de Groot \& Lamers 1992; de Groot et al. 2001).

In this paper we present the analysis of a unique set of new photometric measurements of $\mathrm{P}$ Cyg covering almost two complete decades, combined with archival data from the literature.

\section{The data}

\subsection{Pre-1982 data}

The data for the years 1902-1907 are from von Prittwitz (1907). The observations were done with a Zöllner-type photometer (see Zöllner 1861; Sterken \& Staubermann 2000); the comparison star was BD +36 $3955=29$ Cyg, a $\lambda$ Boo star in the $\delta$ Scuti instability strip with a pulsation amplitude of 0 . 03 in $V$ and $P \sim 45 \mathrm{~min}$. The resulting $m_{v}$ values were not corrected to the $V$ scale. The $m_{v}$ light curve in Fig. 1 shows four blocks of data, totalling 38 measurements. The mean light level is $m_{v}=5.04$ The associated standard deviation $(0.11)$ is rather high, and reflects the uncertainties inherent in visual photometry. There is a rather strong increase in brightness level between the first group and the following ones; the overall brightness gradient is about $0 \mathrm{~m}^{\mathrm{m}} 02 \mathrm{y}^{-1}$ over almost $2000 \mathrm{~d}$. The only structure visible is a maximum around JD 2415898 , and another one around JD 2416700 (Fig. 1).

A second set of early data was published by Nikonov (1937, 1938): 65 data points, $B$ filter close to Johnson $B$, the standard deviation $(0.035)$ is low, and there is a steady increase of the brightness level by $\sim 0{ }^{\mathrm{m}} 03 \mathrm{y}^{-1}$ over the 800 days spanning the observations. Figure 2 gives the $B$ light curve for the recorded measurements. Two clearlydelined light maxima are present, viz. JD 2428074.8 and 2428442 .

A third set is by Groeneveld (1944), and consists of $55 \mathrm{~V}$ measurements collected over a time interval of about five months. Most remarkable is the fast decline in light starting on JD 2431007 , and lasting for three subsequent nights with a gradient of $-0 . \mathrm{m}^{\mathrm{m}} 023 \mathrm{~d}^{-1}$. These data are illustrated in Fig. 3.

Percy \& Welch (1983) published $11 \mathrm{~V}$ measurements of P Cyg (Fig. 4), apparently, a light maximum occurred close to JD 2445123.7 (June 1982).

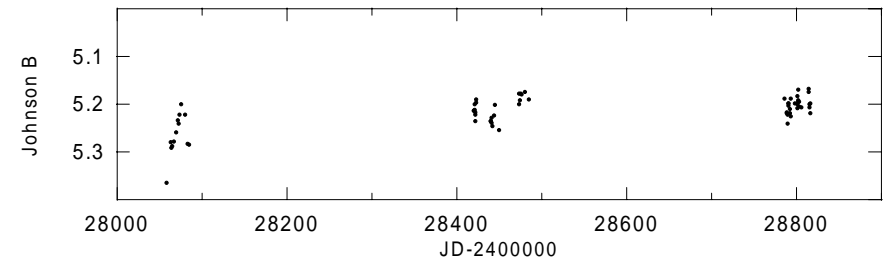

Fig. 2. B light curve of P Cyg, data from Nikonov $(1937,1938)$.

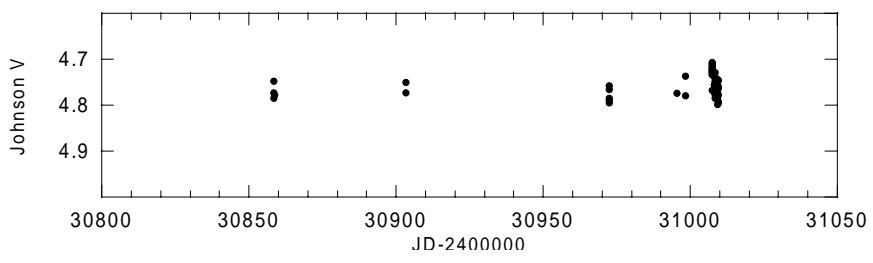

Fig. 3. $V$ light curve of P Cyg, data from Groeneveld (1944).

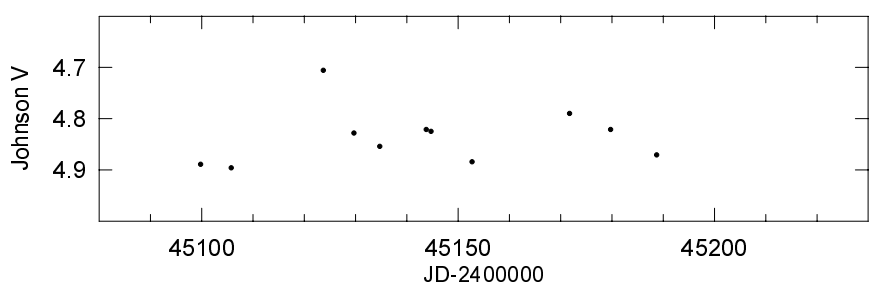

Fig. 4. $V$ light curve of $\mathrm{P}$ Cyg, data from Percy \& Welch (1983).

Table 1. Sources of the post-1982 data, ID is the identification label used in the discussion, $N$ denotes the number of measurements. APT stands for Automatic Photometric Telescope.

\begin{tabular}{lll}
\hline ID & $N$ & Reference \\
\hline$P$ & 38 & Percy et al. (1988) \\
$V R I$ & 95 & From VRI APT data \\
$A P T A$ & 515 & Armagh programme on the APT \\
$A P T L$ & 127 & Leiden programme on the APT \\
$C A M C$ & 118 & Carlsberg Automatic Meridian Circle \\
$D B$ & 17 & Dietmar Böhme, Nessa, Germany \\
$M i$ & 95 & R. Milton, Somes Bar, CA \\
$Z S$ & 31 & E. Zsoldos, Konkoly Observatory Budapest \\
$P S$ & 8 & Peter Sterzinger, Australia \\
$M T$ & 11 & Markova \& Tomov (1998) \\
\hline
\end{tabular}

\subsection{Post-1982 data}

The post-1982 dataset is a combination of $V$ data from different sources, as is described in Table 1. The so far unpublished photometric data used in this study will be submitted for publication to the Journal of Astronomical Data (JAD, 2001). Evidently, one may not anticipate that all these data can be swiftly merged into one composite light curve: the various equipment, sites, observers and comparison stars must inevitably lead to inhomogeneities and systematic errors. In order to minimize such effects, we have attempted to determine a transformation from 

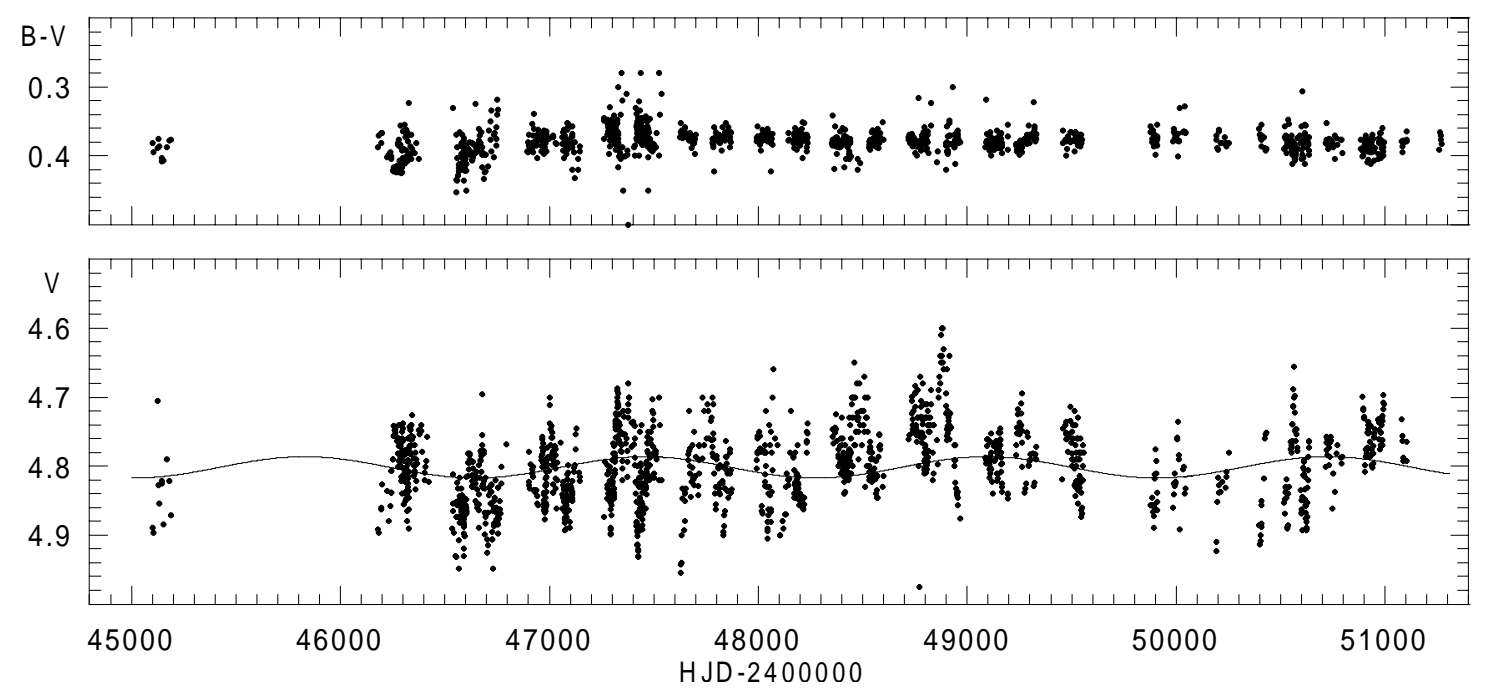

Fig. 5. Combined $V$ light curve. The continuous line represents the best-fitting sine curve with $P=1630$ days and amplitude 0. 015 (fit on the basis of all data collected after JD 2446000).

Table 2. Differences (APTA minus other) of quasisimultaneous observations. $N$ is the number of such data avaiable, $\Delta$ is the average difference, $\sigma$ is s.d.

\begin{tabular}{|c|c|c|c|}
\hline ID & $N$ & $\Delta$ & $\sigma$ \\
\hline$V R I$ & 15 & -0.040 & $0.051^{*}$ \\
\hline$A P T L$ & 44 & -0.010 & 0.011 \\
\hline$C A M C$ & 33 & $0 .{ }^{\mathrm{m}} 044$ & 0.030 \\
\hline$D B$ & 4 & -0.043 & 0.015 \\
\hline$M T L$ & 5 & -0.031 & 0.008 \\
\hline$M i$ & 13 & $0 . \mathrm{m} 015$ & 0.021 \\
\hline$Z S$ & 8 & -0.023 & 0.029 \\
\hline
\end{tabular}

* After removal of erroneous measurements.

each data set to the largest data subset, which is APTA. We point out that the APTA data were obtained using 32 Cyg as comparison star and 22 Cyg as check star, whereas for the APTL data, $22 \mathrm{Cyg}$ was the comparison star, and 32 Cyg the check star.

We searched all data sets of Table 1 for measurements that were obtained within 0.5 from an observation of group APTA. Table 2 gives the result.

It is clear that system $V R I$ yielded the largest instabilities, followed by $C A M C$. For most other datasets the application of a single zero-point shift $(\Delta)$ yields an internally consistent sequence of $V$ magnitudes that are in accord with the data in APTA. Especially for the $V R I$ data, it appeared that the difference is not constant in time.

In order not to degrade any APTA data by quasisimultaneous data coming from other groups, we have used those other data only whenever no APTA data were available. In this way, we obtained the overall 1982-1999 light and colour curves displayed in Fig. 5, and the major part of the same light curve shown in greater detail in Figs. 6 and 7 .
The latter figures illustrate a shape of the light curve of $\mathrm{P}$ Cyg that seems quite characteristic: there is a pseudocyclic behaviour with a characteristic time of the order of 16-19 days. The descending branch after maximum seems to be quite smooth while the rising branch frequently displays some kind of downward bump just preceding maximum - the latter sometimes has the shape of a stillstand as is often seen on the same location in the light curve of Mira-like variables, although pronounced depresssions (looking like small minima) are seen too.

Figure 5 reveals - besides short-term variability on time scales of $16-19 \mathrm{~d}$ and $\sim 100 \mathrm{~d}-\mathrm{a}$ strong and almost cyclic fluctuation of the mean brightness level, with a characteristic time of about $1500-1600 \mathrm{~d}$ or 4 years. Note that the fitted curve (almost 3 cycles) was obtained on the basis of data taken after JD 2446000 only. This long-term fluctuation probably can be identified with the S Dor phases, which are typical for these stars and have a more or less cyclical appearance. Note that, considering the residuals around the sine curve, the cycle of $4 \mathrm{y}$ is not quite unambiguous, see Sect. 6 point 3. In addition, an underlying long-term brightness increase with gradient $0.007 \mathrm{y}^{-1}$ is also present.

\subsection{Hipparcos data}

The Hipparcos catalogue lists 154 reliable $H_{\mathrm{p}}$ magnitudes obtained between JD 2447859 and 24449046. Only 10 of these measurements have been made within half a day from our data. The broad-band $H_{\mathrm{p}}$ magnitudes, as is well known, are based on a very broad passband. Hence a correction is needed to bring the $H_{\mathrm{p}}$ magnitudes to the same scale as our ground-based data. The average Hipparcos magnitude is $4.8674 \pm 0.0016$, whereas the corresponding ground-based data yield an average $V=4.8043 \pm 0.0014$, hence a correction of the $H_{\mathrm{p}}$ magnitudes with $-0{ }^{\mathrm{m}} 063$ is in order. Although these Hipparcos magnitudes do not add 

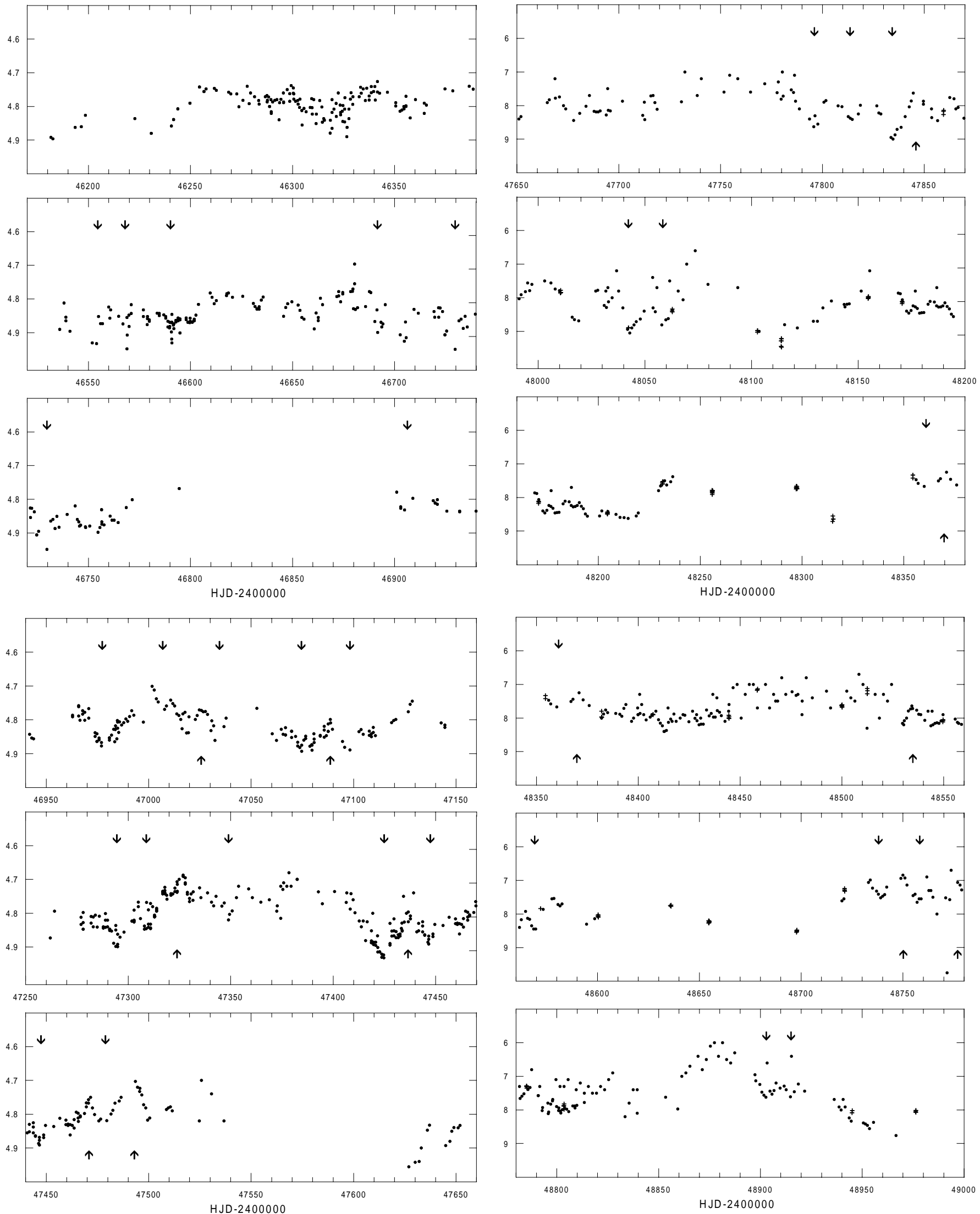

Fig. 6. $V$ light curve of P Cyg $(\bullet)$, times of maximum (Table 4) are indicated by upward arrows, times of minimum (Table 3) by downward arrows. Adjusted Hipparcos magnitudes are represented by + .

Fig. 7. $V$ light curve of P Cyg. 
a single cycle to the data we present here, they are most useful in filling gaps between observing sequences.

\section{Frequency analysis of the post-1982 data}

A Fourier frequency analysis of the data shown in Fig. 5 (omitting the Percy \& Welch data) $)^{1}$ was carried out in the frequency range 0.0001-0.075 cycles-per day $\left(\mathrm{cd}^{-1}\right)$ using the Period software (Sperl 1998). The amplitude spectrum and the spectral window are given in Fig. 8. Almost no power is visible in the period range $15-50$ days (see Sect. 4), but there appear a number of strong peaks at frequencies below $0.006 \mathrm{~cd}^{-1}$, the strongest being at $0.00030 \mathrm{~cd}^{-1}(P=\sim 3100 \mathrm{~d})$ with a corresponding maximum in the spectral window, the second and thirdstrongest at $0.0057(P=175 \mathrm{~d})$ and $0.0020(P=500 \mathrm{~d})$, respectively. The group of the $100 \mathrm{~d}$-type variations is represented by peaks between 0.011 and $0.014 \mathrm{~cd}^{-1}$. A peak at $0.0006 \mathrm{~cd}^{-1}(P=1600 \mathrm{~d})$ seems to correspond to the period of the wave-like pattern seen in Fig. 5.

\section{The pulsation period of P Cyg}

Independently of the Fourier analysis, we determined the basic period of P Cyg using the classical method of establishing an ephemeris on the basis of times of photometric maximum (hereafter indicated by $T_{\max }$ ) and minimum $\left(T_{\min }\right)$. Therefore, we have selected by visual inspection all well-observed extrema in Figs. 6 and 7. With well-observed extrema we understand those groups of data that consist of at least four measurements in the 5-day time interval centered on the visually estimated time of extremum, with the additional condition that a $T_{\min }$ or $T_{\max }$ should not be dominated by one single outlying measurement.

An important element in this approach is the establishment of the cycle-count scheme. A first inspection of the light curves shows that there are five blocks of almost contiguous cycles in which there is virtually no doubt as to the relative cycle-count patterns internal to each block: JD 46 906-47095, 47 294-47479, 47629-47834, 4873848915 and 49145-49517. A linear ephemeris fitted to $T_{\min }$ for each of these groups yields, respectively $P=$ $16.79,18.83,16.96,17.85$ and 17.68 , thus a mean $P=$ $17^{\mathrm{d}} 6 \pm 0.4$. Therefore, we tried to extend the cycle-count scheme to all time intervals falling in between the determined $T_{\min }$ and $T_{\max }$. During this procedure, we also used other indications pointing to the presence of maxima and minima (such as less-well observed extrema).

From a number of pronounced minima, we derived a preliminary ephemeris, and then we determined the cycle number $E$ for all obtained $T_{\min }$; the zeropoint for $E$ is arbitrary, but chosen in such a way that we deal with positive $E$-numbers only. The resulting ephemeris

\footnotetext{
1 These data were omitted because of their isolated character and because they cannot be rigorously transformed to the photometric system in which the other data have been obtained.
}

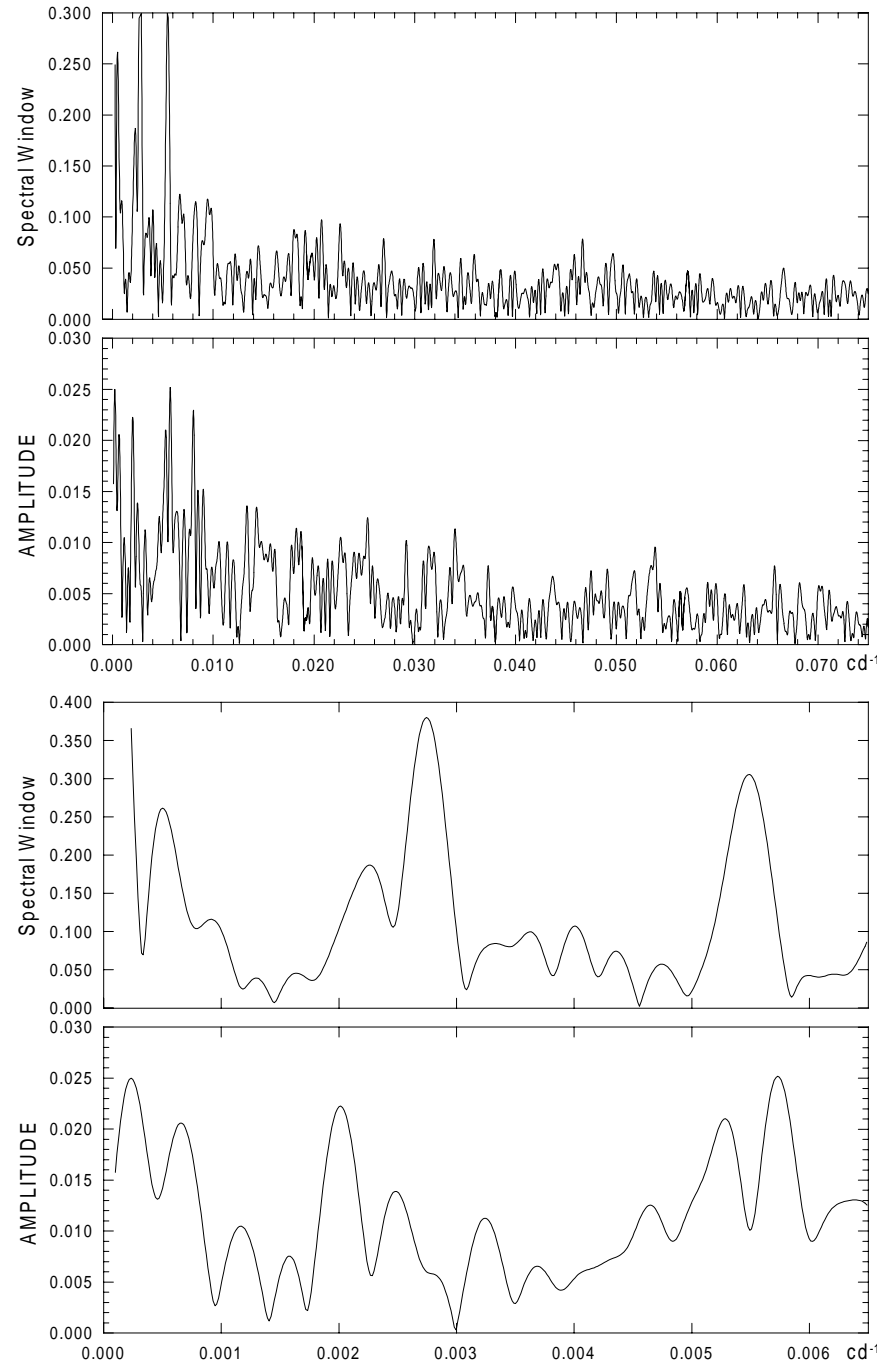

Fig. 8. Frequency spectra of the light curve of P Cyg (19821999). The lower and upper panels give the same information at different frequency resolution.

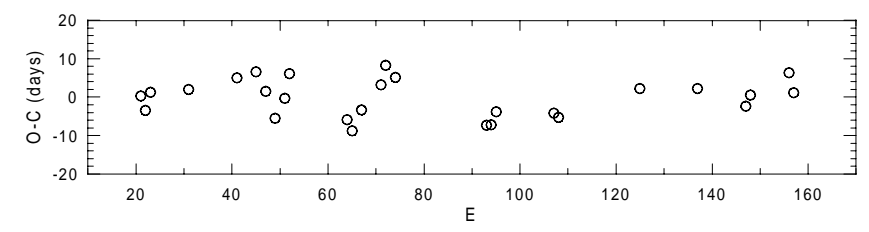

Fig. 9. O-C diagram for all $T_{\min }$ data listed in Table 3 .

turned out to be

$$
\begin{gathered}
\mathrm{HJD}_{\min }=2446189.9+17.34 \mathrm{E} \\
\pm 1.4 \pm 0.1
\end{gathered}
$$

The list of $T_{\min }, E$ values is given in Table 3. Figure 9 gives the resulting $\mathrm{O}-\mathrm{C}$ diagram. Note that this $17.3 \mathrm{cy}-$ cle $\left(0.0576 \mathrm{c} \mathrm{d}^{-1}\right)$ is absent in the amplitude spectrum of Fig. 8.

In a way similar to the one followed in the previous Section, we derived the ephemeris

$$
\begin{gathered}
\mathrm{HJD}_{\max }=2446199.5+17.35 E \\
\pm 6.5 \pm 0.2 .
\end{gathered}
$$


Table 3. Heliocentric times of minimum light of $\mathrm{P}$ Cyg and cycle number $E$.

\begin{tabular}{rrrrrr}
\hline$T_{\min }$ & $E$ & $T_{\min }$ & $E$ & $T_{\min }$ & $E$ \\
\hline 46554.6 & 21 & 47098.2 & 52 & 48042.2 & 107 \\
46568.1 & 22 & 47294.5 & 64 & 48058.4 & 108 \\
46590.2 & 23 & 47308.9 & 65 & 48360.9 & 125 \\
46729.7 & 29 & 47349.0 & 67 & 48569.1 & 137 \\
46906.3 & 41 & 47424.9 & 71 & 48569.1 & 137 \\
46977.3 & 45 & 47447.4 & 72 & 48738.0 & 147 \\
47006.9 & 47 & 47478.9 & 74 & 48758.2 & 148 \\
47021.3 & 48 & 47796.1 & 93 & 48902.9 & 156 \\
47034.5 & 49 & 47813.6 & 94 & 48915.0 & 157 \\
47074.5 & 51 & 47834.3 & 95 & & \\
\hline
\end{tabular}

Table 4. Heliocentric times of maximum light of $\mathrm{P}$ Cyg and cycle number $E$.

\begin{tabular}{rrrrrr}
\hline$T_{\max }$ & $E$ & $T_{\max }$ & $E$ & $T_{\max }$ & $E$ \\
\hline 47025.6 & 47 & 47470.9 & 74 & 48534.8 & 135 \\
47088.6 & 51 & 47493.0 & 75 & 48750.1 & 147 \\
47323.8 & 65 & 47845.9 & 95 & 48776.8 & 148 \\
47436.5 & 71 & 48369.8 & 125 & & \\
\hline
\end{tabular}

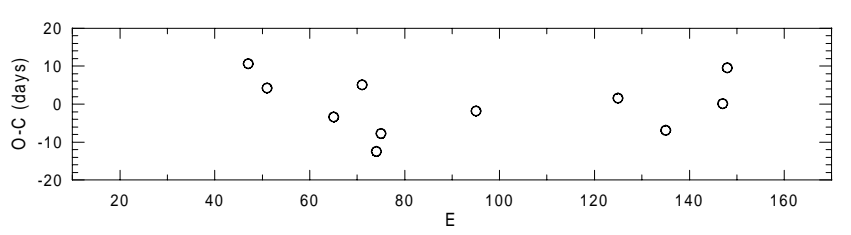

Fig. 10. O-C diagram for all $T_{\max }$ data listed in Table 4.

The list of $T_{\max }, E$ is given in Table 4 . Figure 10 gives the resulting $\mathrm{O}-\mathrm{C}$ diagram which, just like Fig. 9, illustrates that the observed time of extremum can strongly deviate from the calculated value, although no significant trends (linear or polynomial) are present.

\section{Colour variations}

As a first indication of the nature of the photometric variations, it is clearly useful to obtain some insight into P Cygni's colour variations. From a visual inspection of the light and colour curves one obtains the impression that $B-V$ increases (i.e. the star reddens) when $V$ decreases (i.e. when the star brightens). To check this behaviour more objectively, we proceed as follows: Treating data from each observing season (which happens to coincide with the calendar year) separately, we calculate the average value of $B-V$ in each 0 . 02 -wide bin of $V$ (i.e. $4.900>V \geq 4.880 ; 4.880>V \geq 4.860$, etc.), plot the mean value of the $V$ measurements in each bin against the average $B-V$ values, fit a straight line using a simplified least-squares method, and determine the slope $\mathrm{d} V / \mathrm{d}(B-V)$ of the resulting straight line for each year. Every year shows a slope $\mathrm{d} V / \mathrm{d}(B-V)$ between -5 and -11 , implying that a decrease in $V$, i.e. when the star brightens, produces an increase in $(B-V)$, i.e. the star reddens. Bearing in mind that this refers to values of $V$ and $(B-V)$ over a year, we conclude that the above-mentioned variations with time scales between 60 and 130 days are best identified with the so-called $100 \mathrm{~d}-$ type micro-variations often found in S Dor variables near maximum brightness (van Genderen et al. 1997a,b).

Because the amplitude of the 17-day variations is smaller, the resultant smaller variations in $(B-V)$ and the dispersion in the $V$ versus $(B-V)$ relation make the results of an analysis along the above lines more unreliable. Visual inspection of the detailed light and colour curve shows that the colour behaviour in the maxima and minima differs from extremum to extremum: sometimes it is a local maximum (i.e. redder), sometimes it is a local minimum (i.e. bluer), and sometimes it is neither. This is not entirely similar to the colour behaviour of the $\alpha$ Cygnitype variations in other stars (e.g. van Genderen et al. $1997 \mathrm{a}, \mathrm{b})$, where the colour is always bluer in the light maxima.

\section{Discussion}

In a number of aspects, $\mathrm{P}$ Cygni is an exceptional case amongst the S Dor variables. During the last two decades (1982-1999) it showed the two types of microvariations $(\sim 0.1$ and $\sim 0.2)$ on top of a very weak S Dor variability $\left(\sim 0 .{ }^{\mathrm{m}} 1\right)$. In what follows we discuss these three types of instabilities:

1. The microvariability $(\sim 0,1)$ shows a quasi-period of 17.3 that is remarkably stable over 6.5 years. Yet, strictly speaking, one cannot call this a "period" because the individual cycle lengths usually hover between 10 and $25 \mathrm{~d}$, apart from the highly-variable shape of the cycles. The total absence of a significant single peak at the frequency of the 17.3 period in the amplitude spectrum is, therefore, understandable. Normally, cycle length variations of this type of microvariation (called the $\alpha$ Cyg-type by van Genderen et al. 1997a) are much smaller, resulting in at least a marginally significant peak in the amplitude spectrum. The weak-active S Dor variable R 85 in the LMC is to some degree similar to $\mathrm{P}$ Cyg considering its mixture of oscillations of different duration (van Genderen et al. 1998), while the normal $\alpha$ Cyg variable (thus no S Dor variable) WRA 977 also shows a stable quasiperiod like P Cyg, over a time interval of 17 years (van Genderen \& Sterken 1996). The stability of the 17.3 oscillation of P Cyg is reflected by the linear ephemeris for the epochs of its extrema. Obviously, its engine is periodic, but its effect on the photospheric light is of a highly quasi-periodic character. One could speculate that this is due to travel-time disturbances from the source in the interior to the surface by a stronger and longer time-scale rolling wave moving there and back. 
Some systematic behaviour in the photospheric response due to the 17.3 oscillations is suspected because of a certain pattern in the $\mathrm{O}-\mathrm{C}$ diagrams, but the evidence is still weak (de Groot et al. 2001). With this quasi-period, $\mathrm{P}$ Cyg fits very well the grid of $P=$ constant lines for $\alpha$ Cyg variations in the $\mathrm{H}-\mathrm{R}$ diagram (van Genderen \& Sterken 1996);

2. The long-term presence of the $17^{\mathrm{d}} 3$ oscillations on top of the 100d-type microvariations $(\sim 0.2)$ is outstanding. Only two other S Dor variables showed this, but only for a few months (Sect. 1.6 in van Genderen 2001). Could the quasi-periodicity of the shorter one be caused by the longer one? We did not find any relationship between the $\mathrm{O}-\mathrm{C}$ values and the place within the 100 d-type cycles.

These 100 d-type cycles show a highly variable duration as well: between $60 \mathrm{~d}$ and $130 \mathrm{~d}$, but this is not abnormal. Israelian et al. (1996) suggested a correlation between the visual changes to a maximum (using part of the photometry presented here) and the ejection of recurrent shells deduced from the so-called discrete absorption components (DACs) as seen in IUE spectra. They estimated the frequency of the shells between $150 \mathrm{~d}$ and $250 \mathrm{~d}$. However, this could well be an upper limit since the IUE observations show large gaps in time series. The analysis by van Gent \& Lamers (1986), on the other side, revealed $60-70 \mathrm{~d}$ but this was also based on scattered spectrocopy.

Markova et al. (2001a) found an anti-correlation between these 100 d-type oscillations and the equivalent width of the $\mathrm{H} \alpha$ emission line. Kolka (1999) discussed a 100 d-type cyclicity in the main spectroscopic features due to moving opacity enhancements (the DACs). He suggested as causes expanding density shells, or - inspired by the fact that the probable rotation period is roughly $100 \mathrm{~d}$ - corotating spiral density waves in the wind. We presume that the 100 d-type cyclicity in the spectrum and of the shells is somehow related to the 100 d-type light variations. The relatively long duration of many light maxima (also $\sim 100 \mathrm{~d}$ ) rather suggests that we are dealing with a global or nearly global phenomenon, rather than with say, a large corotating hot spot. The long duration of the maxima is presumably an argument against an origin in the wind.

Since a light variation of $\sim 0.2$ means a variation of $\sim 20 \%$ in $L$ (assuming that the complete energy distribution varies more or less by the same amount), the 100 d-type light variations could be caused by a radius change of $10 \%$ (at constant $T$ ) in case of a radial pulsation, or by a $T$ variation of $5 \%$ (at constant radius, thus no radial pulsation). The latter case is not likely because of the inconsistency with the observations (red colour in the maxima), see further.

Could they be explained by a larger than $10 \%$ increase of the radius and with a simultaneous slightly decreasing $T$ and vice versa? This would imply a mean radial velocity variation of the photosphere amounting to $\sim 1 \mathrm{kms}^{-1}$ adopting $R=75 R_{\odot}$ (Najarro et al. 1997). This effect is too small to be detectable. If P Cygni were much cooler, one could suspect that the source of such a pulsational behaviour could be the low-amplitude $(\sim 0.2$ in $L$ !) oscillations due to the $\kappa$ mechanism found by Stothers \& Chin $(1995,1996)$ and Stothers $(1999 b, 2000)$ in their models for yellow supergiants, called relaxation oscillations, with a time scale of months! It has been speculated before that the 100 d-type light variations, usually present around the maxima of the S Dor phases, when the star is coolest, could be related to these oscillations (van Genderen 2001). However, in view of P Cygni's high temperature, the relaxation oscillations would have been much shorter, perhaps in the order of days.

The bi-stability mechanism proposed by Pauldrach \& Puls (1990) is able to explain some of the observed characteristics of the shell ejections, while the light variation should then only be due to photospheric $T$ variations by backscattering from the shell, i.e. $T$ increases if the high mass-loss episode starts and vice versa. However, the light maxima should then become bluer, while the opposite is observed. An explanation for this inconsistency might be offered by the fact that shells are denser than the wind and if cooler than the photosphere, could weaken to some extent the flux at the shorter wavelengths relative to the longer ones.

But how does this shell creation start in the first place? We hypothesize that the cause is the source of the 100 d-type light oscillations. After all, P Cygni's wind is - according to Pauldrach \& Puls' (1990) model very unstable with respect to small changes in the luminosity $(>3 \%)$ and/or the radius $(>1.5 \%)$ of the star;

3. The very weak S Dor cycles - between 1982 and 1999 - have a range of $\sim 00^{\mathrm{m}} 1$ on a time-scale of $\sim 4$ y. It is quite possible that the observations in the nineteen nineties represent a cycle about twice as long (Fig. 5 and de Groot et al. 2001). Support for this longer one is given by Markova et al. (2001a, 2001b). They show that the $\mathrm{H} \alpha$ emission line is possibly cyclic on a time-scale of that order $(\sim 7 \mathrm{y})$ and in phase with the brightness: maxima in 1985 (JD 2446300 ) and 1992 (JD 2448800 ), and minima in 1988 (JD 2447500) and in 1995 (JD 2450000$)$. Besides, they demonstrate that it probably represents an S Dor phase as tentatively suggested by van Genderen (2001). They find that during the light maximum the radius increased by $7 \%$ and the temperature decreased by $10 \%$. It should be noted that the SD cycles need not be periodic. Consecutive cycles can appreciably differ in duration and range from cycle to cycle, see e.g. R 71 in Sterken et al. (1997b) and other S Dor variables (van Genderen et al. 1997a, 1997b).

The simultaneous $\mathrm{H} \alpha$ spectroscopy by Markova et al. (2001a, 2001b) suggests that the short (S)-SD phase (short, because it is <10 y, van Genderen 2001) is likely 
accompanied by changes in the properties of the wind, i.e. an increase in the mass-loss rate and a decrease in the velocity field. These conclusions are based on the relative large optical depth of the wind, which led them to conclude that P Cyg likely has a permanent pseudo-photosphere with $\tau_{\nu}=2.30$.

\section{Conclusion}

$\mathrm{P}$ Cygni is subject to at least three very-well documented types of instabilities during the last two decades of the 20th century: a short-SD cyclicity $(<10 \mathrm{y})$, albeit a very weak active one and the well-known two types of microvariations. Considering the small variations in the scattered observations until the beginning of the 18th century and even further back in time, led de Groot et al. (2001) to speculate that the SD variability is going on since the eruption in the 17 th century.

The $\alpha$ Cygni-type microvariations show a remarkably stable quasi-period of $17^{\mathrm{d}} .3$; the other type of microvariation shows the typical 100-d cyclicity with usually redder colours in the maxima. We speculate that the DACs with a time scale of months and the recurrent shell ejections, together with the photometric 100 d-type variations, are related with each other, because they all have roughly the same time scale. The 100 d-type light variations can then be explained by the variations of the physical parameters of the star while the colours are influenced by opacity changes in the wind due to recurrent shells. Wellobserved S Dor variables with large amplitudes show near minimum brightness the $\alpha$ Cygni-type, and near maximum the 100 d-type oscillations. Halfway between the two extrema the one is quickly replaced by the other, probably within a few months (e.g. van Genderen et al. 1990, 1997b). Both can only be seen simultaneously during this short transition stage. Therefore, the continuous presence of the latter two in the case of $\mathrm{P}$ Cyg is quite exceptional.

Acknowledgements. CS acknowledges a research grant from the Belgian Fund for Scientific Research (NFWO). Research at the Armagh Observatory is grant-aided by the Department of Culture, Arts and Leisure for Northern Ireland, and by the UK PPARC through the provision of the STARLINK network.

\section{References}

de Groot, M. 1969, BAN, 20, 225

de Groot, M., \& Lamers, H. J. 1992, Nature, 355, 422

de Groot, M., Sterken, C., \& van Genderen, A. M. 2001, in P Cygni 2000, 400 years of progress, ed. M. de Groot, \& C. Sterken, ASP Conf. Ser., 233, 15

van Genderen, A. M. 1991, in Wolf-Rayet stars and interrelations with other massive stars in galaxies, ed. K. A. van der Hucht, \& B. Hidayat (Kluwer), 563 van Genderen, A. M. 2001, A\&A, 366, 508

van Genderen, A. M., \& Sterken, C. 1996, A\&A, 308, 763

van Genderen, A. M., Thé, P. S., Heemskerk, M., et al. 1990, A\&AS, 82, 189

van Genderen, A. M., van de Bosch, F. C., Dessing, F., et al. 1992, A\&A, 264, 88

van Genderen, A. M., Sterken, C., \& de Groot, M. 1997a, A\&A, 318,81

van Genderen, A. M., de Groot, M., \& Sterken, C. 1997b, A\&AS, 124, 517

van Genderen, A. M., Sterken, C., \& de Groot, M. 1998, A\&A, 337,393

van Gent, R. H., \& Lamers, H. J. G. L. M. 1986, A\&A, 158, 335

Groeneveld, I. 1944, Veröff. Landessternwarte Heidelberg, 14

Israelian, G., \& de Groot, M. 1999, Sp. Sci. Rev., 90, 493

Israelian, G., de Groot, M., Parker, W., \& Sterken, C. 1996, MNRAS, 283, 119

de Jager, C. 2001, in P Cygni 2000, 400 years of progress, ed. M. de Groot, \& C. Sterken, ASP Conf Ser., 233, 215

Kolka, I. 1999, in Variable and non-spherical Stellar Winds in Luminous Hot Stars, IAU Coll., 169, ed. B. Wolf, O. Stahl, \& A. W. Fullerton (Springer), 268

Markova, N. 2000, A\&AS, 144, 391

Markova, N. 2001, in P Cygni 2000, 400 years of progress, ed. M. de Groot, \& C. Sterken, ASP Conf. Ser., 233, 95

Markova, N., \& Tomov, N. 1998, IBVS, 4641

Markova, N., Morrison, N., Kolka, I., \& de Groot, M. 2000, ASP Conf. Ser., 204, 111

Markova, N., Scuderi, S., de Groot, M., Markov, H., \& Panagia, N. 2001a, A\&A, 366, 935

Markova, N., Morrison, N., Kolka, I., \& Markov, H. 2001b, in press

Najarro, F., Hillier, D. J., \& Stahl, O. 1997, A\&A, 326, 1117

Nikonov, V. 1937, Bull. Abastuman. Obs., 1, 35

Nikonov, V. 1938, Bull. Abastuman. Obs., 2, 23

Pauldrach, A. W. A., \& Puls, J. 1990, A\&A, 237, 409

Percy, J. R., \& Welch, D. L. 1983, PASP, 95, 491

Percy, J. R., Napke, A. E., Richer, M. G., et al. 1988, A\&A, 191, 248

Prittwitz, F. 1907, AN, 4210

Sperl, M. 1998, software made available at ftp://dsn.astro.univie.ac.at/pub/Period98/

Sterken, C., \& Staubermann, K. 2000, in Karl Friedrich Zöllner and the historical dimension of astrophotometry, ed. C. Sterken, \& K. Staubermann, VUBPRESS, Brussels

Sterken, C., de Groot, M., \& van Genderen, A. M. 1997a, A\&A, 326,640

Sterken, C., van Genderen, A. M., \& de Groot, M. 1997b, ASP Conf. Ser., 120, 35

Sterken, C., de Groot, M., \& van Genderen, A. M. 1998, A\&A, 333,565

Stothers, R. B. 1999a, ApJ, 516, 366

Stothers, R. B. 1999b, MNRAS, 305, 365

Stothers, R. B. 2000, personal communication

Stothers, R. B., \& Chin Chao-wen 1995, ApJ, 451, L61

Stothers, R. B., \& Chin Chao-wen 1996, ApJ, 468, 842

Zöllner, K. F. 1861, Grundzüge einer allgemeinen Photometrie des Himmels (Berlin) 